\title{
Mucosal correlates of isolated HIV semen shedding during effective antiretroviral therapy
}

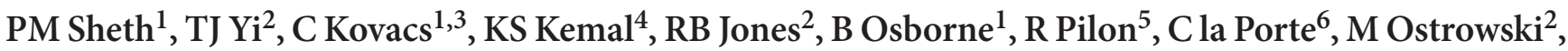 \\ T Mazzulli $^{7,8}$, H Burger $^{4}$, B Weiser ${ }^{4}$ and R Kaul ${ }^{1,2,9}$
}

Effective antiretroviral therapy (ART) suppresses the blood HIV RNA viral load (VL) below the level of detection. However, some individuals intermittently shed HIV RNA in semen despite suppression of viremia, a phenomenon termed "isolated HIV semen shedding (IHS)". In a previously reported clinical study, we collected blood and semen samples from HIV-infected men for 6 months after ART initiation, and documented IHS at $\geq 1$ visit in almost half of the participants, independent of ART regimen or semen drug levels. We now report the mucosal immune associations of IHS in these men. Blood and semen plasma cytokine levels were assayed by multiplex enzyme-linked immunosorbent assay, T-cell populations were evaluated by flow cytometry in freshly isolated blood and semen mononuclear cells, and semen cytomegalovirus (CMV) DNA levels were measured by PCR. Although IHS was not associated with altered blood or semen cytokine levels, the phenomenon was associated with a transient, dramatic increase in CD4 + and CD8 + T-cell activation that was restricted to the semen compartment. All participants were CMV infected, and although semen CMV reactivation was common despite ART, this was not associated with T-cell activation or IHS. Further elucidation of the causes of compartmentalized mucosal T-cell activation and IHS may have important public health implications.

\section{INTRODUCTION}

Sexual transmission accounts for $>80 \%$ of human immunodeficiency virus (HIV) infections globally. ${ }^{1}$ The blood HIV RNA viral load (VL) predicted the transmission risk within HIV serodiscordant couples in whom the index case was not taking antiretroviral therapy (ART), ${ }^{2}$ probably because the blood VL generally correlates with virus levels in both cervicovaginal secretions ${ }^{3}$ and semen. ${ }^{4}$ However, a direct import of virus from blood into semen is not the only contributor to the genital tract VL, and there also seems to be oligoclonal amplification of virus in the genital tract and in compartmentalized semen virus sources. ${ }^{5}$ In keeping with this, both the semen and vaginal VL each predicted HIV sexual transmission risk independently of the blood VL in HIV-serodiscordant couples. ${ }^{6}$

Effective ART suppresses the blood VL below the limit of detection, and ART often profoundly reduces the semen HIV RNA load as well. ${ }^{7-10}$ This means that ART has the potential to substantially reduce the sexual transmission of HIV at a population level. ${ }^{11}$ An observational study demonstrated a $92 \%$ reduction in HIV transmission in HIV serodiscordant couples after the seropositive partner started ART, ${ }^{12}$ and a randomized clinical trial of ART to prevent transmission demonstrated a $96 \%$ reduction; ${ }^{13}$ however, occasional transmission occurred in both studies despite ART. A significant subset of men on effective ART continue to shed HIV RNA in semen, ${ }^{7,8,14-16}$ and a similar phenomenon is observed in the female genital tract. ${ }^{17}$ The degree to which this phenomenon of "isolated semen HIV shedding" (IHS) contributes to residual HIV transmission despite effective ART remains to be studied. However, elucidating the causes of IHS may be very important from a public health perspective.

Other than the blood VL, several other factors have been associated with a higher HIV RNA level in the semen of ART-naïve men. These factors include semen cytomegalovirus (CMV)

\footnotetext{
${ }^{1}$ Department of Medicine, University of Toronto, Toronto, Ontario, Canada. ${ }^{2}$ Department of Immunology, University of Toronto, Toronto, Ontario, Canada. ${ }^{3}$ Maple Leaf Medical Clinic, Toronto, Ontario, Canada. ${ }^{4}$ Wadsworth Center, New York State Department of Health, Albany, New York, USA. ${ }^{5}$ Center for Communicable Diseases and Infection Control, Public Health Agency of Canada, Ottawa, Ontario, Canada. ${ }^{6}$ The Ottawa Hospital and Ottawa Health Research Institute, University of Ottawa, Ottawa, Ontario, Canada. ${ }^{7}$ Laboratory Medicine and Pathobiology, University of Toronto, Toronto, Ontario, Canada. ${ }^{8}$ Department of Microbiology, Mount Sinai Hospital, Toronto, Ontario, Canada. 9University Health Network, Toronto, Ontario, Canada. Correspondence: PM Sheth (prameet.sheth@utoronto.ca) and R Kaul (rupert.kaul@utoronto.ca) 
reactivation, ${ }^{18}$ herpes simplex type 2 seropositivity, ${ }^{19} \mathrm{com}$ partmentalized viral quasispecies, ${ }^{20}$ higher semen HIV levels in therapy-naïve men, ${ }^{8}$ and increased semen inflammatory cytokine and chemokine levels ${ }^{5,21,22}$ that may enhance mucosal T-cell activation and virus replication. ${ }^{5}$ In addition, antiretroviral agents demonstrate highly variable semen penetration, with much lower levels of efavirenz and several protease inhibitors in semen compared with blood, ${ }^{23,24}$ but with equivalent or higher semen levels of agents such as 3TC, raltegravir, and maraviroc. $^{25-27}$ This suggests that the selection of different antiretroviral regimens might alter the propensity to IHS, although this has not been well studied.

We previously described a cohort of men starting ART in whom we prospectively collected paired blood and semen samples for 6 months. Almost half $(12 / 25 ; 48 \%)$ of the participants had at least one visit with IHS despite an undetectable blood VL, and of the participants with IHS a third (4/12) had high levels of semen viral RNA ( $>5,000$ copies per $\mathrm{ml}) .{ }^{8}$ In that study, IHS was not associated with standard clinical parameters (blood VL or CD4 count), antiretroviral regimen (protease inhibitors vs. non-nucleoside reverse transcriptase inhibitors), semen drug levels, the presence of drug resistance mutations or herpes simplex type 2 serostatus. However, IHS during ART was associated with a higher semen HIV VL before therapy initiation. ${ }^{8}$ We have now expanded these studies to assess the association of IHS with systemic and semen immune parameters, as well as with CMV reactivation in the semen compartment. We observed a dramatic, compartmentalized immune activation of semen CD4 + and CD8 + T cells during IHS episodes, which was independent of classical sexually transmitted infections (STIs) and of semen CMV reactivation or cytokine/chemokine levels. This suggests that IHS is driven by a compartmentalized inflammatory process, and that elucidating the cause of this immune activation could lead to novel ways to prevent HIV transmission.

\section{RESULTS}

\section{Frequency of IHS}

Participant demographics and the frequency of isolated HIV semen shedding (IHS) have been reported previously. ${ }^{8}$ In brief, $25 \mathrm{HIV}$-infected therapy-naive individuals were enrolled, with paired blood and semen samples collected at baseline (before therapy initiation) and at weeks 2, 4, 8, 12, 16, 20, and 24 of therapy.

\section{Characteristics of viral isolates during IHS episodes}

As previously published, no resistance mutations to the three major drug classes were detected in semen viruses during high-level IHS episodes. ${ }^{8}$ Phylogenetic tree analysis including reference strains in the Los Alamos HIV Sequence Database (http://www.hiv.lanl.gov/content/sequence/HIV/mainpage. $\mathrm{html}$ ) was performed for all baseline plasma and semen isolates, and for IHS isolates from three individuals with highlevel HIV RNA ( $>5,000$ copies per $\mathrm{ml}$ of semen plasma). All the sequences were unique and of subtype B origin (data not shown), and enrolment (pre-ART) sequences derived from the blood plasma and semen of each patient were closely related but distinct. There was no evidence of super-infection or recombination. Variant analysis of semen sequences was performed for participant OM304 in the pre-ART semen sample (data not shown), and phylogenetic tree analysis of the virus from his IHS episode (OM304S-506) clustered closely with one of these semen variants. V3 sequence analysis predicted CCR5 tropism for the semen IHS virus.

\section{T-cell activation and semen HIV RNA level before starting ART}

Cervical CD4 + T-cell activation, as defined by expression of the early activation marker CD69, has been correlated with the local HIV RNA levels in the genital compartment of ART-naïve women. ${ }^{28}$ Therefore, we examined its association with the preART semen VL in 23 of 25 participants, of whom 18 of 23 had sufficient semen CD3 + T-cell numbers to permit analysis $(\geq 500$ $\mathrm{CD} 3+\mathrm{T}$ cells). ${ }^{22} \mathrm{~T}$-cell expression of CD69 was much higher in semen than in blood, both on CD4 + T cells (median 17.9 vs. $0.7 \%$, respectively; $P=0.0003$ ) and CD $8+$ T cells (median 25.5 vs. $1.75 \% ; P=0.0005)$. The semen HIV RNA VL was directly correlated with the proportion of semen $\mathrm{CD} 4+\mathrm{T}$ cells expressing CD69 ( $r=0.53, P=0.02$; Figure 1). Although there was a strong positive correlation between the expression of CD69 on semen and blood CD $4+\mathrm{T}$ cells $(r=0.58, P=0.006)$, the semen VL was not associated with blood T-cell activation $(r=0.11$, $P=0.67)$. There was no correlation between the number of $\mathrm{CD} 4+$ or $\mathrm{CD} 8+\mathrm{T}$ cells in semen and the semen VL. Immune activation of blood or semen $\mathrm{CD} 4+$ or $\mathrm{CD} 8+\mathrm{T}$ cells, as defined by their expression of CD69+, did not correlate with cytokine levels in blood or semen.

\section{Compartmentalized T-cell activation in semen during IHS episodes}

As semen T-cell activation had been correlated with the semen VL before starting ART, we then examined T-cell immune alterations during subsequent IHS episodes. At enrolment, participants who went on to have at least one IHS episode $(12 / 25 ; 48 \%)$ did not demonstrate differences in overall semen T-cell numbers (median T-cell numbers IHS vs. no IHS: 2,487 vs. 2,393, $P=0.31$ ) or semen T-cell immune activation (all $P>0.1$ ). Within each participant, we then compared T-cell immune activation at three time points: at baseline (pre-ART), at the IHS visit with the highest semen VL (or a matched time point for those participants without IHS), and at the last study visit where IHS was not detected (Figure 2). IHS episodes were not associated with any immune activation alterations in blood CD4 + or CD8 + $T$ cells (Figure 2a and $\mathbf{b}$, respectively). However, they were associated with a dramatic increase in the proportion of semen T cells expressing CD69; this was apparent on both semen CD $4+\mathrm{T}$ cells (median $27.4 \%$ baseline vs. $51.8 \%$ IHS; $P=0.016$; Figure 2c) and CD8 + T cells (median 19.9\% baseline vs. 58.8\% IHS; $P=0.004$; Figure 2d). After the IHS episode, the proportion of T cells expressing CD69 decreased to baseline in both CD4+ and CD $8+$ T cells $(P=0.008$ and $P=0.040$ for the reduction after IHS, respectively; Figure $2 \mathbf{c}$ and d). This transient increase in 


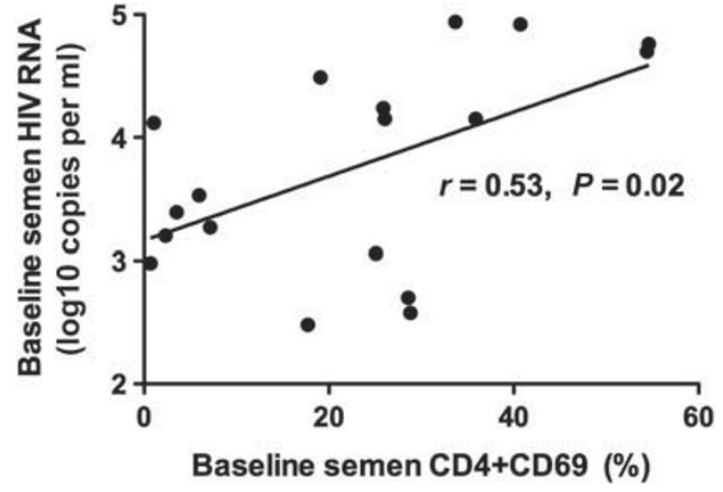

Figure 1 Pre-ART semen CD4 + T-cell activation and the semen viral load. In ART-naïve participants, a positive correlation was observed between the semen $V L$ and percentage expression of immune activation marker CD69 + on semen CD4 + T cells. ART, antiretroviral therapy; VL, viral load.

semen T-cell activation was limited to participants with IHS, and no alterations in T-cell activation were seen in the blood (data not shown) or semen (Figure $2 \mathbf{e}$ and $\mathbf{f}$ ) of participants without IHS.

Immune activation during IHS episodes only manifested as an increase in the proportion of semen T cells expressing CD69, and there was no corresponding increase in overall semen T-cell numbers, the CD69 + mean fluorescence intensity, or in the absolute number of activated T cells. In particular, there were similar numbers of total semen mononuclear cells, CD3 +, $\mathrm{CD} 4+$, and CD8 + T cells at baseline and during episodes of IHS (all $P>0.4$; data not shown), as well as during and after resolution of IHS (all $P>0.4$; data not shown). In the subset of participants with insufficient cell numbers to permit analysis $(<500 \mathrm{CD} 3+\mathrm{T}$ cells $)$, the frequencies of observed IHS were similar $(P=0.5)$.

\section{Semen cytokine/chemokine levels and IHS}

IHS was associated with semen T-cell activation as assayed by flow cytometry, and the semen VL in ART-naïve men has been associated with increased pro-inflammatory semen cytokines. ${ }^{22}$ Therefore, we measured levels of interleukin (IL)-1b, IL-6, IL-7, IL-8, IL-10, monocyte chemotactic protein-1 (MCP-1), interferon gamma-induced protein-10, monokine induced by interferon- $\gamma$, and regulated upon activation normal T-cell expressed and secreted (RANTES) in blood and semen plasma in all individuals with available samples (23/25 participants). Cytokine levels were measured at baseline (pre-ART), at an intermediate time point when IHS was detected (or a matched time point for participants with no IHS), and at the last study visit where IHS was not detected at baseline (pre-ART). At baseline, semen levels of IL-1b (range: $0-10.1 \mathrm{pg} \mathrm{ml}^{-1}$ ) and IL-10 (range: $0-27.6 \mathrm{pg} \mathrm{ml}^{-1}$ ) were consistently below the limit of assay detection, whereas those of MCP-1 (range: $18->8,000 \mathrm{pg} \mathrm{ml}^{-1}$ ) frequently fell above the dynamic range of the assay.

Pre-ART (enrolment) semen cytokine concentrations did not differ between participants with or without subsequent
IHS (Figure 3a-f), and unexpectedly the blood levels of IL-6, RANTES, and IL-8 tended to be lower in those participants who went on to develop IHS (Figure 3a-c). Prospective analysis of semen cytokine and chemokine levels demonstrated an initial decrease in semen levels IL-8 after ART initiation, both for participants with and without IHS, and a subsequent increase to levels higher than those seen before ART (Figure 4a and $\mathbf{b}$ ). No significant changes were seen in semen MCP-1 (Figure 4e and f), IL-6, IP10, or IL-7 (Figure $\mathbf{4 g}$ and $\mathbf{h}$ ) levels in either group. Only RANTES (Figure $\mathbf{4 c}$ and d) levels differed significantly by IHS status; unexpectedly, a transient decrease in semen RANTES levels was seen during IHS episodes, and no corresponding alteration was seen in participants without IHS (Figure $4 \mathrm{c}$ and $\mathbf{d}$ ).

\section{CMV infection, semen CMV reactivation, and IHS}

All study participants were CMV IgG seropositive, and semen $\mathrm{CMV}$ reactivation has been associated with a disproportionately high semen HIV VL in therapy-naïve men. ${ }^{18}$ Therefore, we measured semen CMV DNA levels during all 168 of 200 (84\%) study visits with sufficient seminal plasma. CMV DNA was detected in the seminal plasma of 13 of 25 (52\%) individuals at the baseline (pre-ART) visit, and in 15 of $25(60 \%)$ participants during at least one study visit. Overall, ART initiation had no impact on semen CMV levels (Figure $\mathbf{5 c}$ and $\mathbf{d}$ ). In those participants with semen CMV DNA detected before ART, CMV levels remained unchanged at 1 month after ART initiation $(P=0.48)$ and after 6 months of ART $(P=0.44)$. Neither the detection of semen CMV DNA before ART initiation nor the level of semen CMV DNA at baseline was associated with subsequent IHS (Figure 5a). Furthermore, longitudinal analysis of semen CMV levels in participants with IHS demonstrated no differences in semen CMV DNA pre-ART, during IHS episodes, and at time points when semen VL was undetectable on suppressive ART (Figure 5b). Semen CMV DNA levels were similar at baseline and after 6 months of suppressive ART, both in participants without IHS and those who demonstrated IHS at one visit or more (Figure 5a). Semen CMV reactivation at the baseline (pre-ART) visit was associated with elevated levels of several semen cytokines including RANTES (mean: 1,295 vs. $772 \mathrm{pg} \mathrm{ml}^{-1}, P=0.009$ ), IL-7 (341 vs. $192 \mathrm{pg} \mathrm{ml}^{-1}, P=0.02$ ), and MCP-1 (5,289 vs. $\left.2,695 \mathrm{pg} \mathrm{ml}^{-1}, P=0.02\right)$, although these associations were not seen after ART initiation. Overall, we were unable to demonstrate any association between semen CMV reactivation and the occurrence of IHS in men taking ART.

\section{DISCUSSION}

Effective ART substantially reduces HIV transmission at a population level, ${ }^{12,29}$ but whether it can completely abrogate transmission is not clear. ${ }^{30,31}$ We along with others ${ }^{7,8,14-16}$ have observed that a small but significant proportion of individuals on effective ART continue to shed HIV RNA in semen, a phenomenon that we refer to as IHS. Although the impact of IHS on HIV transmission is not known, the phenomenon is of great public health interest and a better understanding of clinical and immune correlates of IHS may serve to inform 
Participants with IHS
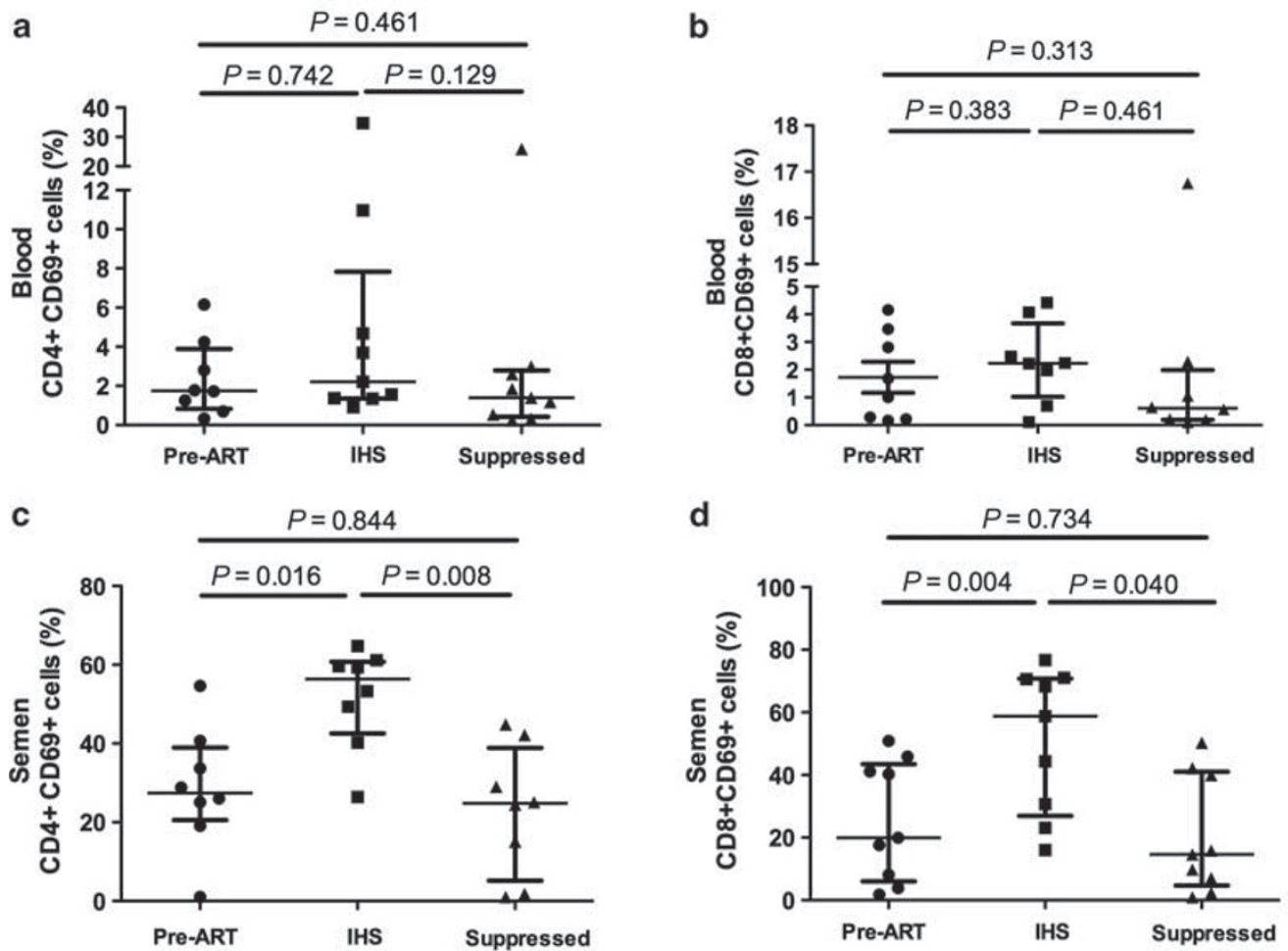

Participants without IHS
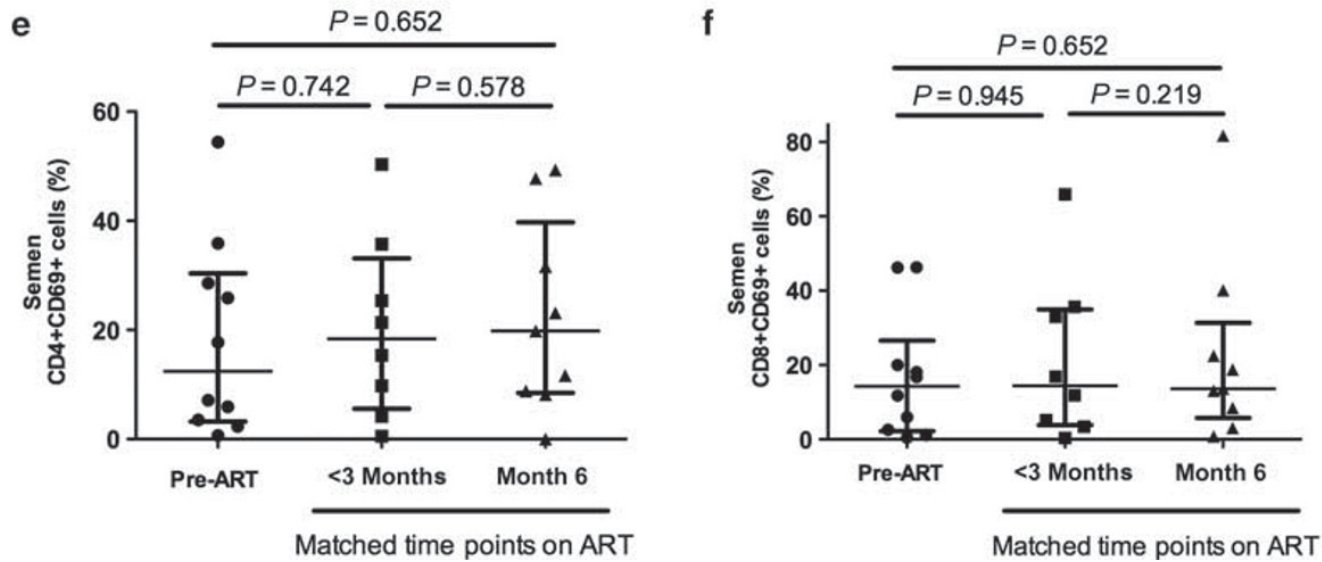

Figure 2 Association of IHS episodes with compartmentalized semen T-cell activation. Expression of the early activation marker CD69 was examined on CD4 + and CD8 + T cells at baseline (pre-ART), at the IHS visit with the highest semen VL (or a matched time point for participants with no IHS), and at the last study visit where IHS was not detected in blood (a and $\mathbf{b}$ ) and semen (c- $\mathbf{f})$. IHS episodes were associated with a dramatic increase in T-cell activation that was limited to the semen compartment (panels $\mathbf{c}$ and $\mathbf{d}$ ). There was no difference in CD69 expression levels at CD4+ or CD8 + T cells at baseline and any other visit in participants with no observed IHS (panels e and f). ART, antiretroviral therapy; IHS, isolated HIV semen shedding; VL, viral load.

strategies to reduce HIV transmission. Partly based on factors that were associated with an increased HIV semen VL in ARTnaïve men, we have now investigated the mucosal immune correlates of IHS using blood and semen samples collected during a previously published clinical study that examined IHS after the initiation of effective ART. ${ }^{8}$ IHS episodes were associated with a dramatic increase in $\mathrm{T}$-cell activation that was limited to the semen compartment, but not with increases in cytokine/chemokine levels in the semen or blood, semen reactivation of CMV, classical STIs, or HIV super-infection/ recombination.

HIV preferentially replicates within activated CD4 + T cells, and T-cell immune activation is associated with an increased VL in the blood, ${ }^{32}$ gastrointestinal mucosa, ${ }^{33}$ and genital tract. ${ }^{22,28}$ 

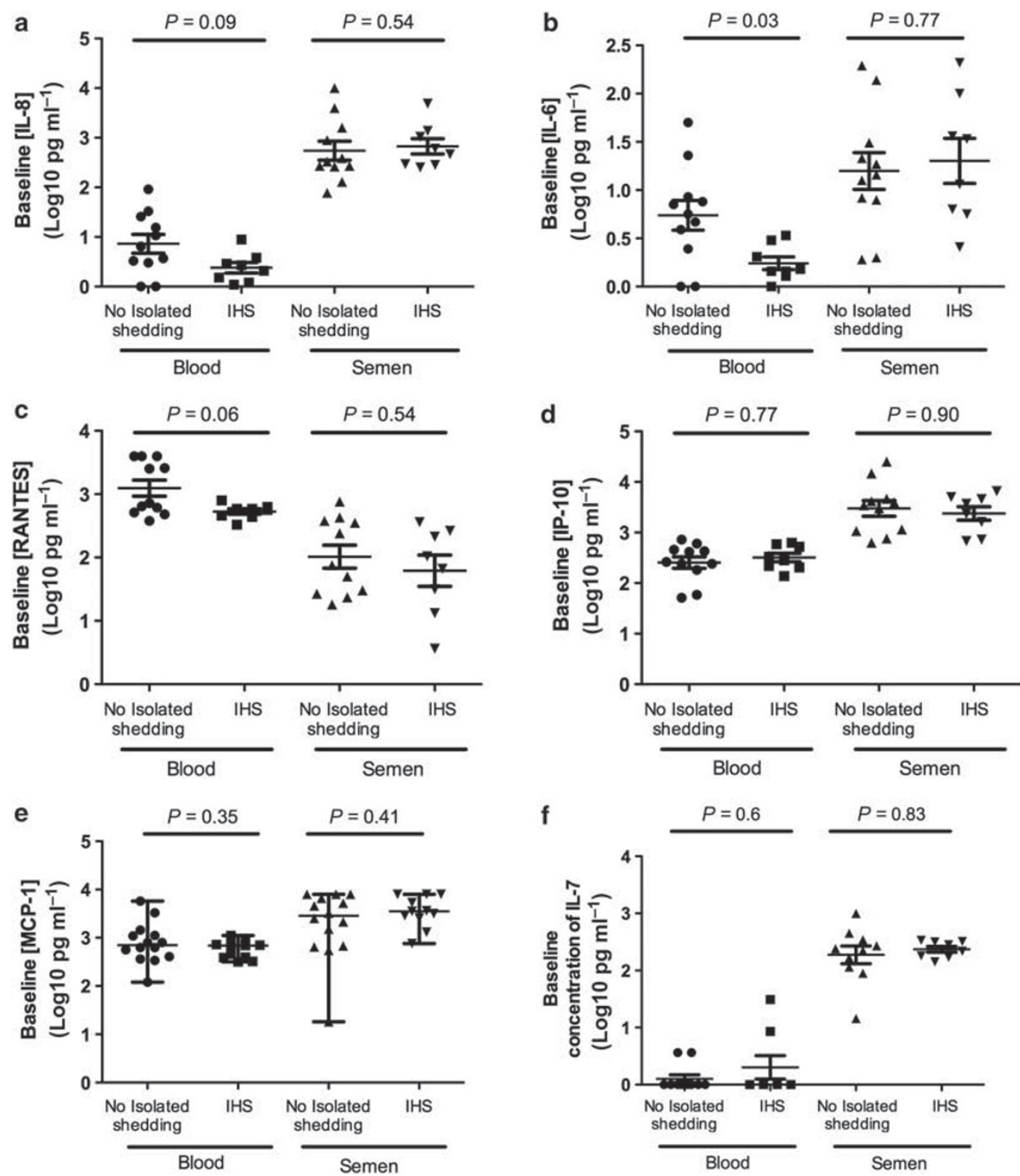

Figure 3 Pre-ART cytokine levels and subsequent IHS. Cytokine levels in blood (left two columns) and semen (right two columns). Pre-ART semen pro-inflammatory cytokine (IL-6, IL-8, IP-10; a, b, d) and chemokine (RANTES, MCP-1; c and e) levels did not differ between participants with or without subsequent IHS. Blood cytokine levels of IL-6 (panel b) were lower in participants with subsequent IHS. In addition levels of blood IL-8 (panel a) and RANTES (panel c) tended to be lower during episodes of IHS compared with pre-ART levels. ART, antiretroviral therapy; HIV, human immunodeficiency virus; IHS, isolated HIV semen shedding; IL, interleukin; IP-10, interferon gamma-induced protein-10; MCP-1, monocyte chemotactic protein-1; RANTES, regulated upon activation normal T-cell expressed and secreted; VL, viral load.

The fact that we observed a dramatic, transient increase in semen T-cell activation that was limited to participants with IHS clearly demonstrates that the phenomenon of IHS is real, although the cause of this increased mucosal activation was not clear. Our observational study is not able to determine the direction of causation, and it is possible that the local HIV reactivation itself was the cause of semen T-cell immune activation. However, numerous previous studies have shown that factors associated with increased genital HIV levels in HIV-infected women, including genital herpes and other infections, cause increased mucosal T-cell activation even in HIV-uninfected individuals. ${ }^{28,34,35}$ This suggests that mucosal T-cell activation is the primary event, and that in HIV-infected individuals, this then leads to local increases in HIV replication. The cause of this compartmentalized T-cell activation during IHS episodes was not clear in our study, and lack of association with classical STIs or CMV reactivation means that more research is required, both to determine the cause of this phenomenon and to develop strategies for prevention. 

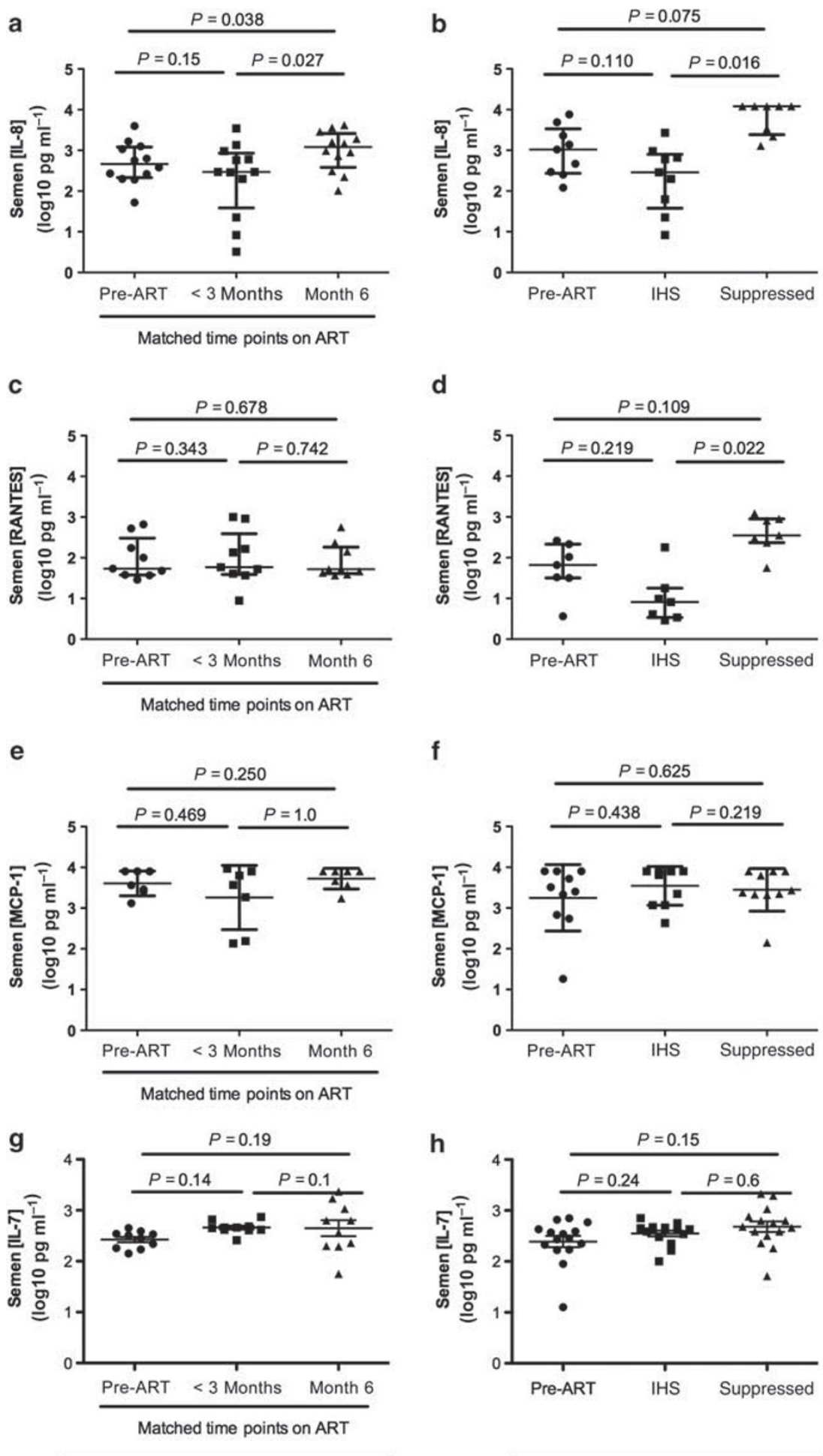

Participants without IHS

Participants with IHS

Figure 4 IHS episodes and semen cytokine levels. Semen and blood levels of several cytokines/chemokines were assessed longitudinally. Similar patterns were seen for participants with/without IHS (IL-8, a and b; MCP-1, e and f). Semen RANTES levels were transiently decreased during IHS, with no corresponding alteration in participants without IHS (RANTES, $\mathbf{c}$ and d). ART, antiretroviral therapy; IHS, isolated HIV semen shedding; IL, interleukin; MCP-1, monocyte chemotactic protein-1; RANTES, regulated upon activation normal T-cell expressed and secreted; VL, viral load. 

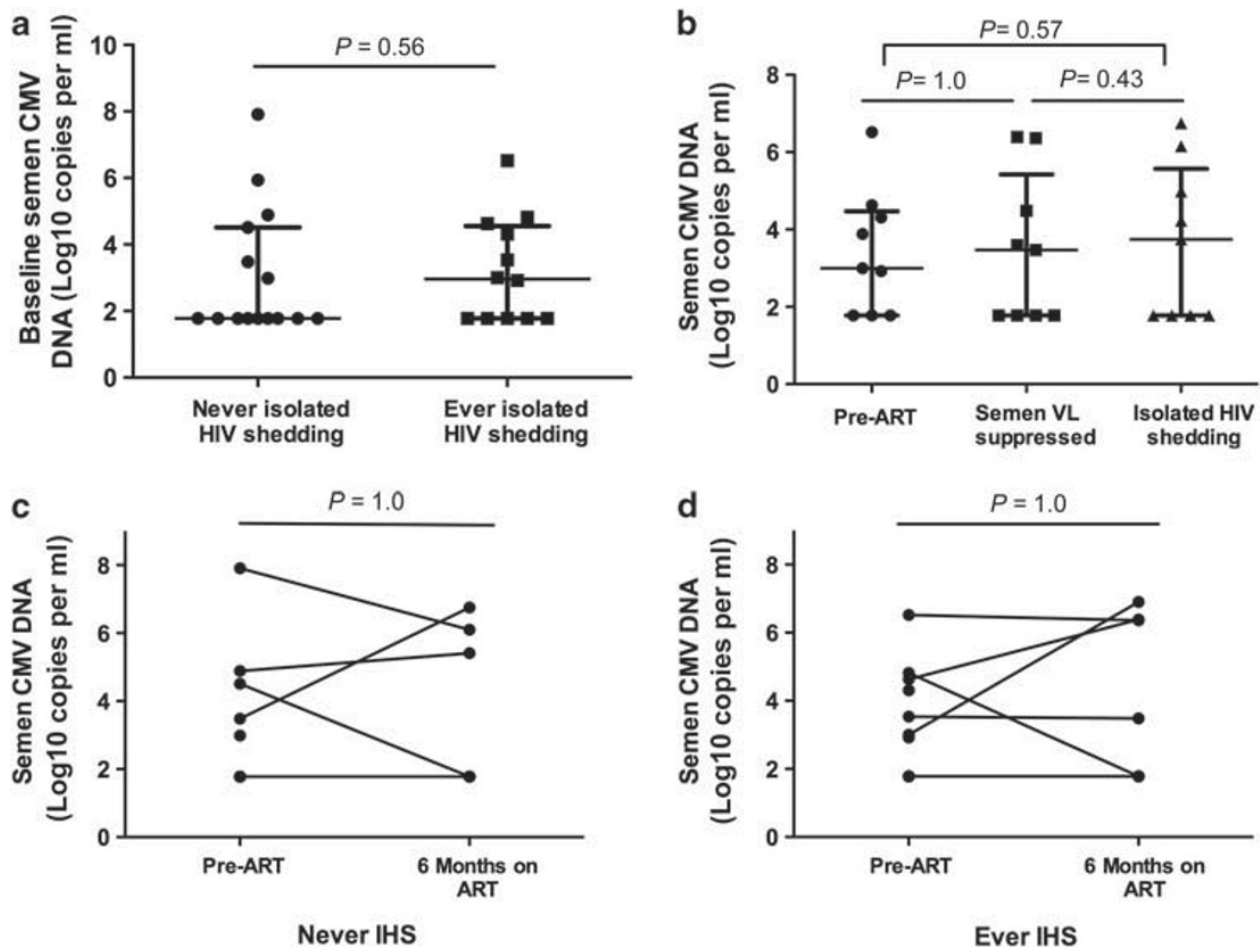

Figure 5 Lack of association between semen CMV reactivation and IHS. (a) Baseline semen CMV DNA levels were not associated with IHS. (b) Longitudinal analysis found no association between semen CMV levels and IHS episodes. CMV DNA levels were not altered after 6 months of effective ART, either (panel c) in participants without IHS, or (panel d) in those who did demonstrate IHS. ART, antiretroviral therapy; CMV, cytomegalovirus; IHS, isolated HIV semen shedding.

As CMV reactivation and absolute CMV DNA semen levels have been associated with a disproportionately high semen HIV VL in ART-naïve men, ${ }^{18}$ we carefully evaluated the association of semen CMV reactivation and CMV DNA levels with IHS. All our study participants were CMV infected, and 60\% shed CMV on at least one study visit. Interestingly, effective ART had no detectable impact on the frequency of CMV reactivation, and we saw no association between CMV reactivation or CMV DNA levels and the phenomenon of IHS.

We previously found that semen pro-inflammatory cytokine levels were associated with increased semen HIV RNA in ARTnaive men, ${ }^{22}$ and so we assayed cytokine levels at baseline, during episodes of IHS and while completely suppressed in both blood and semen. Our multiplex cytokine enzyme-linked immunosorbent assay seemed to provide robust and reproducible results, and the semen/blood cytokine ratios observed were generally comparable to those reported by Anderson et al..$^{5}$ (data not shown). We saw no clear association of IHS with increased blood or semen pro-inflammatory cytokine or chemokine levels, although we observed a trend to elevated semen IL-6 levels in individuals with IHS $(P=0.07)$. Surprisingly, semen RANTES levels actually decreased during episodes of IHS. These findings were somewhat unexpected, as semen pro-inflammatory cytokines had been associated with HIV levels in ART-naïve men. ${ }^{22,34}$ The reason for this is not clear, although the latter studies were performed in therapy-naive individuals who were not expected to start ART for at least a year and had much less severe immune compromise. ${ }^{22}$ It is possible that the association of IHS with lower RANTES levels may relate to increased internalization of this chemokine into activated $\mathrm{T}$ cells, a phenomenon that has been previously reported during HIV RNA replication in vitro. ${ }^{36}$

Most semen virus in men without urethritis or prostatitis originates in the lower urogenital tract, distal to the prostate, ${ }^{37}$ and both local oligoclonal virus amplification and compartmentalized semen sources contribute to the semen VL. ${ }^{5}$ The transient nature of IHS episodes and the strong association with compartmentalized semen T-cell activation make it tempting to hypothesize that an undefined urethral pathogen may have been responsible for IHS. Indeed, in previous studies ART-naive men with a disproportionately high semen VL reported having had higher rates of unprotected insertive (but not receptive) sex, suggesting the possibility of an undefined urethral pathogen in this context as well. ${ }^{38}$ We did not screen for T. vaginalis or M. genitalium, both of which can cause urethritis in men, but the lack of clinical symptoms or increased urine leukocytes on dipstick make these diagnoses unlikely.

Our cohort sample size and the duration of follow-up after ART initiation were relatively limited, but the large effect size that we demonstrated means that the described mucosal immune associations of IHS are very robust. All our study participants were on either protease inhibitor or non-nucleoside (NNRTI)-based therapy, ${ }^{8}$ both of which penetrate poorly into the semen. ${ }^{23,27}$ Raltegravir (an integrase inhibitor) and 
maraviroc (an R5 inhibitor) demonstrate improved semen penetration, ${ }^{25,26}$ and so the impact of such regimens on IHS incidence may merit further study.

We hypothesize that the phenomenon of IHS may have real world significance. Two important recent studies demonstrated that ART initiation was associated with a dramatic $92-96 \%$ reduction in heterosexual HIV transmission. ${ }^{12,13}$ The rare transmission events that were seen in these studies all occurred soon after ART initiation, the period during which our study was focused. Before ART initiation, we detected high-level semen HIV shedding ( $>5,000$ HIV RNA copies per ml) in just over half of our participants (52\%); after suppression of the blood HIV VL, we found high-level IHS at 5 of 119 visits (4.2\%). This $92 \%$ reduction in high-level HIV shedding correlates closely with the actual reduction in HIV transmission events that was seen in recent clinical studies. ${ }^{12,13}$ However, determining whether high-level IHS is causally associated with rare HIV transmission events occurring soon after starting ART will require larger clinical studies that specifically focus on this question.

In summary, we have expanded our original description of IHS in a subset of men starting effective ART to explore the clinical and immune associations of this phenomenon. IHS was associated with dramatic immune activation of CD4+ and CD8 + T cells that was limited to the semen compartment. Although this might suggest an infectious cause for IHS, the phenomenon was not associated with classical STIs, urine leukocytes by dipstick, or with semen CMV reactivation. Further research into the cause of IHS and its public health implications is required, and may help to improve the effectiveness of ART as an HIV prevention tool.

\section{METHODS}

Ethics statement. All participants provided informed, written consent and the study protocol was approved by the Research Ethics Board at the University of Toronto (Toronto, Canada).

Study participants and design. HIV-infected men were recruited through an HIV primary care clinic (the Maple Leaf Medical Clinic, Toronto, Canada). ${ }^{8}$ Participants had no evidence of urethritis or other active STIs at baseline. All HIV-infected men were therapy-naïve, with a detectable blood HIV RNA VL before starting therapy. Simultaneous blood and semen samples were collected at baseline and then after ART initiation prospectively at weeks $2,4,8,12,16,20$, and 24 after ART initiation. The ART regimens were selected by individual physicians, based on current clinical guidelines, ${ }^{39}$ and the results of baseline antiretroviral resistance screening (VirtualPhenotype, Virco, Bridgewater, NJ).

Specimen collection and diagnostics. Blood was collected into acid citrate dextran and semen samples were collected by masturbation into a sterile container containing $10 \mathrm{ml}$ RPMI with penicillin/streptomycin. ${ }^{22}$ Blood and semen plasma were isolated by centrifugation, and HIV RNA load measured using the Versant HIV-1 RNA 3.0 assay (bDNA; Bayer Diagnostics, Puteaux Cedex, France). Seminal plasma VLs (and immune parameters, see below) were multiplied by an appropriate dilution factor to correct for dilution during sample collection, as described previously. ${ }^{18,22}$ A semen VL > 5,000 RNA copies per ml was defined as "high-level" shedding, as the semen VL tends to be $\sim 10$-fold lower than blood, ${ }^{22,40}$ and individuals with a blood VL $>50,000$ RNA copies per $\mathrm{ml}$ are more likely to transmit. ${ }^{2} \mathrm{~A}$ first-void urine sample was screened for infection by Neisseria gonorrhea or Chlamydia trachomatis (Amplicor CT/NG assay, Roche Diagnostics, QC, Canada), and syphilis serology was performed. Blood CMV serology was performed using the human CMV IgG (AxSYM CMV IgG assay, Abbott Laboratories, Abbotts Park, $\mathrm{IL}$ ) and semen CMV VL was measured using real-time PCR (lower limit of detection, 10 DNA copies per ml: Artus) on a Roche Lightcycler (Roche Diagnostics).

HIV sequence analysis and genotyping. RNA was extracted from blood/semen plasma using the Total RNA Isolation System Kit (Promega, Madison, WI), ${ }^{41}$ and cDNA was synthesized using real time-PCR and the SuperScript III first-strand synthesis system (Invitrogen, Carlsbad, CA). ${ }^{42}$ A 1.6-kb fragment encompassing the protease and reverse transcriptase regions of HIV-1 pol was amplified by using nested PCR primers and the XL PCR kit containing rTth DNA polymerase enzyme (Applied Biosystems, Foster City, CA). ${ }^{43}$ Population-based sequences were obtained for samples with low VLs $(<5,000$ copies per $\mathrm{ml})$. For samples with high viral copies, sequences were obtained for multiple, unique, variants. To determine unique variants, serial four-fold cDNA dilutions were performed and each dilution was PCR amplified. The end point dilution was defined as the last dilution of cDNA yielding a positive PCR. Multiple aliquots of the end point dilution were subjected to amplification, yielding detectable PCR products in $30-40 \%$ of the aliquots. Both strands of the PCR products were then sequenced for each unique variant. Computational analysis was performed by using BioEdit software (http://www.mbio.ncsu.edu/BioEdit/bioedit.html) for sequence alignment, and MEGA version 4.0 (http://www.megasoftware.net) for phylogenetic analyses. ${ }^{43}$ Phylogenetic trees were constructed after gap stripping of columns and using the neighbor-joining method.

Where no HIV-1 RNA was amplified from semen plasma using this protocol, the Nuclisens EasyMag System (BioMerieux, St Laurent, QC, Canada) was used. ${ }^{44}$ Viral nucleic acids were lysed in $2 \mathrm{ml}$ of buffered guanidium thiocyanate for $10 \mathrm{~min}$ at RT, and then silica-bound nucleic acids were washed and eluted into a low-salt buffer. Protease and RT regions were amplified using an in-house nested reverse transcription-PCR method, ${ }^{45}$ with amplification in two overlapping pieces of $\sim 700$ bases using One-Step reverse transcription-PCR (Qiagen, Mississauga, ON, Canada) under standard conditions $\left(35\right.$ cycles, $53^{\circ} \mathrm{C}$ annealing) and custom primers. Nested PCR was performed using AmpliTaq Gold (ABI, Foster City, CA) with $2 \mathrm{mM} \mathrm{MgCl}_{2}$ (35 cycles, $53^{\circ} \mathrm{C}$ annealing). Sequences were generated using BigDye v3.1 (ABI) and resolved on an ABI Prism 3130xl analyser. Sequence electropherograms were aligned to HXB2 reference sequence using SeqScape software (ABI) to generate a contiguous sequence. Genotypic drug resistance was determined by submitting the sequences obtained to the Stanford HIV Drug Resistance Database (http://hivdb.stanford.edu).

Cytokine levels in blood and semen plasma. Cytokine levels in blood and semen plasma were measured using the Search Light (Aushon Biosystems, Billerica, MA) protein multi-array system. Nine cytokines/ chemokines were measured in blood and semen plasma, including IL-1b, IL-2, IL-6, IL-7, IL-8, IL-10, RANTES, MCP-1, and monokine induced by IFN- $\gamma$. All assays were run in duplicate at 1:2 and 1:10 dilutions. For cytokine levels that were above the upper limit of detection of the assay, values were reported as the upper limit of detection in the most diluted sample. All samples were assayed according to the manufacturer's protocol. Cytokine concentrations were log transformed for analysis.

T-cell populations in blood and semen. Mononuclear cells were isolated from the blood and semen as described previously. ${ }^{22}$ In brief, peripheral blood mononuclear cells were collected into Acid Citrate Dextran solution A (ACD; BD Bioscience, La Jolla, CA), and semen samples were collected in $10 \mathrm{ml}$ of RPMI media with $100 \mathrm{U} \mathrm{ml}^{-1}$ penicillin, $100 \mathrm{mg} \mathrm{ml}^{-1}$ streptomycin, and 1× GlutaMAX-1 (Gibco, Grand Island, NY) and transported to the laboratory within $3 \mathrm{~h}$ of collection. Mononuclear cells from blood and semen were isolated by layering samples over Ficoll-Paque Plus (Amersham Biosciences, Foster City, CA) and centrifuged at $500 \mathrm{~g}$ 
for 25 min without brakes, counted, and washed twice in RPMI 1640 with $10 \%$ heat-inactivated fetal bovine serum (Sigma, St Louis, MO), $100 \mathrm{U} \mathrm{ml}^{-1}$ penicillin, $100 \mathrm{mg} \mathrm{ml}^{-1}$ streptomycin, and $1 \times$ GlutaMAX-1 (Gibco). Mononuclear cells were then stained for surface markers (CD3APC, CD8-PerCP, CD56-FITC, and CD69-PE) and then analyzed using flow cytometry. CD69+ expression on T cells was used as a marker of short-term immune activation, as in previous studies examining the correlates of HIV shedding in the female genital tract. ${ }^{28} \mathrm{CD} 3+\mathrm{T}$ cells not expressing CD8 were defined as CD4 + T cells.

Statistical analysis. SPSS 12 (SPSS, Chicago, IL) and GraphPad 5 (GraphPad, La Jolla, CA) for Windows were used for statistical analysis. Comparisons between blood and semen were performed using nonparametric Wilcoxon's signed ranks paired $t$-test and the Friedman ranked analysis of variance. Dichotomous variables were compared between groups by $\chi^{2}$ analysis with calculation of likelihood ratios' continuous variables were compared using the Mann-Whitney nonparametric test. Paired analyses were performed using the Friedman non-parametric test.

\section{ACKNOWLEDGMENTS}

We gratefully acknowledge the contributions of all study participants. This work was supported by the Canadian Institutes of Health Research (RK; HOP-81735 and HOP-115020), the Ontario HIV Treatment Network (RK; ROG-B161), and the Canada Research Chair Programme (RK, salary support). Study sponsors had no role in study design, collection or analysis of data, interpretation of results, writing of the manuscript, or decision to submit for publication.

\section{AUTHOR CONTRIBUTIONS}

Study concept: PMS, CK, RK, KSK, HB, BW, and RK. Study design: PMS, CK, RBJ, MO, CLP, RK, KSK, HB, BW, and RK. Clinical/experimental procedures: PMS, TJY, BO, RBJ, CLP, RP, KSK, TM, and RH. Data analysis: PMS and RK. Initial manuscript draft: PMS and RK. Manuscript revisions: CK, KSK, HB, BW, CLP, RBJ, MO, TM, and RK.

\section{DISCLOSURE}

The authors declared no conflict of interest.

C 2012 Society for Mucosal Immunology

\section{REFERENCES}

1. Hladik, F. \& McElrath, M.J. Setting the stage: host invasion by HIV. Nat. Rev. Immunol. 8, 447-457 (2008).

2. Quinn, T.C. et al. Viral load and heterosexual transmission of human immunodeficiency virus type 1. Rakai Project Study Group. N Engl. J. Med. 342, 921-929 (2000).

3. Kovacs, A. et al. Determinants of HIV-1 shedding in the genital tract of women. Lancet 358, 1593-1601 (2001).

4. Xu, C., Politch, J.A., Tucker, L., Mayer, K.H., Seage, G.R. III \& Anderson, D.J. Factors associated with increased levels of human immunodeficiency virus type 1 DNA in semen. J. Infect. Dis. 176, 941-947 (1997).

5. Anderson, J.A. et al. HIV-1 populations in semen arise through multiple mechanisms. PLoS Pathog. 6, e1001053 (2010).

6. Baeten, J.M. et al. Genital HIV-1 RNA predicts risk of heterosexual HIV-1 transmission. Sci. Transl. Med. 3, 77ra29 (2011).

7. Marcelin, A.G. et al. Detection of HIV-1 RNA in seminal plasma samples from treated patients with undetectable HIV-1 RNA in blood plasma. AIDS 22, 1677-1679 (2008).

8. Sheth, P.M. et al. Persistent HIV RNA shedding in semen despite effective antiretroviral therapy. AIDS 23, 2050-2054 (2009).

9. Neely, M.N. et al. Cervical shedding of HIV-1 RNA among women with low levels of viremia while receiving highly active antiretroviral therapy. J. Acquir. Immune Defic. Syndr. 44, 38-42 (2007).

10. Fiore, J.R. et al. Correlates of HIV-1 shedding in cervicovaginal secretions and effects of antiretroviral therapies. AIDS 17, 2169-2176 (2003).

11. Montaner, J.S. et al. The case for expanding access to highly active antiretroviral therapy to curb the growth of the HIV epidemic. Lancet 368 , 531-536 (2006).
12. Donnell, D. et al. Heterosexual HIV-1 transmission after initiation of antiretroviral therapy: a prospective cohort analysis. Lancet $\mathbf{3 7 5}$, 2092-2098 (2010).

13. Cohen, M.S. et al. Prevention of HIV-1 infection with early antiretroviral therapy. N Engl. J. Med. 365, 493-505 (2011).

14. Barroso, P.F. et al. Effect of antiretroviral therapy on HIV shedding in semen. Ann. Intern. Med. 133, 280-284 (2000).

15. Halfon, P. et al. Semen may harbor HIV despite effective HAART: another piece in the puzzle. PLoS ONE 5, e10569 (2010).

16. Lorello, G., la Porte, C., Pilon, R., Zhang, G., Karnauchow, T. \& MacPherson, P. Discordance in HIV-1 viral loads and antiretroviral drug concentrations comparing semen and blood plasma. HIV Med. 10, 548-554 (2009).

17. Cu-Uvin, S. et al. Genital tract HIV-1 RNA shedding among women with below detectable plasma viral load. AIDS 24, 2489-2497 (2010).

18. Sheth, P.M. et al. Disproportionately high semen shedding of HIV is associated with compartmentalized cytomegalovirus reactivation. J. Infect. Dis. 193, 45-48 (2006).

19. Butler, D.M. et al. Herpes simplex virus 2 serostatus and viral loads of $\mathrm{HIV}$-1 in blood and semen as risk factors for HIV transmission among men who have sex with men. AIDS 22, 1667-1671 (2008).

20. Gupta, P. et al. Human immunodeficiency virus type 1 shedding pattern in semen correlates with the compartmentalization of viral Quasi species between blood and semen. J. Infect. Dis. 182, 79-87 (2000).

21. Berlier, W., Bourlet, T., Levy, R., Lucht, F., Pozzetto, B. \& Delezay, O. Amount of seminal IL-1 beta positively correlates to HIV-1 load in the semen of infected patients. J. Clin. Virol. 36, 204-207 (2006).

22. Sheth, P.M. et al. HIV-specific CD8+ lymphocytes in semen are not associated with reduced HIV shedding. J. Immunol. 175, 4789-4796 (2005).

23. Reddy, Y.S. et al. Pharmacokinetic and pharmacodynamic investigation of efavirenz in the semen and blood of human immunodeficiency virus type 1-infected men. J. Infect. Dis. 186, 1339-1343 (2002).

24. Taylor, S. et al. Antiretroviral drug concentrations in semen of HIV-infected men: differential penetration of indinavir, ritonavir and saquinavir. J. Antimicrob. Chemother. 48, 351-354 (2001).

25. Barau, C. et al. High concentration of raltegravir in semen of HIV-infected men: results from a substudy of the EASIER-ANRS 138 trial. Antimicrob. Agents Chemother. 54, 937-939 (2010).

26. Antiretrovirals for prevention: maraviroc exposure in the semen and rectal tissue of healthy male volunteers after single and multiple dosing (paper \#85). Proceedings of the 17th Conference on Retroviruses and Opportunistic Infections (CROI); February 16-19 2010; San Francisco, CA.

27. Kashuba, A.D., Dyer, J.R., Kramer, L.M., Raasch, R.H., Eron, J.J. \& Cohen, M.S. Antiretroviral-drug concentrations in semen: implications for sexual transmission of human immunodeficiency virus type 1. Antimicrob. Agents Chemother. 43, 1817-1826 (1999).

28. Rebbapragada, A. et al. Negative mucosal synergy between herpes simplex type 2 and HIV in the female genital tract. AIDS 21, 589-598 (2007).

29. Attia, S., Egger, M., Muller, M., Zwahlen, M. \& Low, N. Sexual transmission of HIV according to viral load and antiretroviral therapy: systematic review and meta-analysis. AIDS 23, 1397-1404 (2009).

30. Statement: antiretroviral therapy and sexual transmission of HIV, www.who.int/entity/hiv/mediacentre/080201_hivtransmission_en.pdf (2008). Accessed Date Accessed 2008 Accessed.

31. Vernazza, P., Hirschel, B., Bernasconi, E. \& Flepp, M. Les personnes séropositives ne souffrant d'aucune autre MST et suivant un traitement antirétroviral efficace ne transmettent pas le $\mathrm{VIH}$ par voie sexuelle (HIV-positive individuals without additional sexually transmitted diseases and on effective anti-retroviral therapy are sexually non-infectious). Bulletin Des Médecins Suisses 89, 165-169 (2008).

32. Orendi, J.M. et al. Activation and cell cycle antigens in CD4+ and CD8+ T cells correlate with plasma human immunodeficiency virus (HIV-1) RNA level in HIV-1 infection. J. Infect. Dis. 178, 1279-1287 (1998).

33. Sheth, P.M. et al. Immune reconstitution in the sigmoid colon after long-term HIV therapy. Mucosal. Immunol. 1, 382-388 (2008).

34. Kaul, R. et al. The genital tract immune milieu: an important determinant of HIV susceptibility and secondary transmission. J. Reprod. Immunol. 77, 32-40 (2008)

35. Zhu, J. et al. Persistence of HIV-1 receptor-positive cells after HSV-2 reactivation is a potential mechanism for increased HIV-1 acquisition. Nat. Med. 15, 886-892 (2009). 
36. Amara, A. et al. HIV coreceptor downregulation as antiviral principle: SDF-1alpha- dependent internalization of the chemokine receptor CXCR4 contributes to inhibition of HIV replication. J. Exp. Med. 186, 139-146 (1997)

37. Coombs, R.W. et al. Lower genitourinary tract sources of seminal HIV. J. Acquir. Immune Defic. Syndr. 41, 430-438 (2006)

38. Kalichman, S.C. et al. Human immunodeficiency virus in semen and plasma: investigation of sexual transmission risk behavioral correlates. AIDS Res. Hum. Retroviruses 17, 1695-1703 (2001).

39. DHHS. Panel on Antiretroviral Guidelines for Adults and Adolescents. Guidelines for the use of antiretroviral agents in HIV-1-infected adults and adolescents, 1-161 (2009).

40. Sheth, P.M. et al. HIV viral shedding in semen: lack of correlation with systemic virus-specific CD8 responses. AIDS 18, 2202-2205 (2004).
41. Fang, G. et al. Long-term survivors in Nairobi: complete HIV-1 RNA sequences and immunogenetic associations. J. Infect. Dis. 190, 697-701 (2004).

42. Fang, G. et al. Recombination following superinfection by HIV-1. AIDS 18, 153-159 (2004).

43. Kemal, K.S. et al. HIV-1 drug resistance in variants from the female genital tract and plasma. J. Infect. Dis. 195, 535-545 (2007).

44. Bertagnolio, S. et al. HIV-1 drug resistance surveillance using dried whole blood spots. Antivir. Ther. 12, 107-113 (2007).

45. Kemal, K.S. et al. HIV-1 in genital tract and plasma of women: compartmentalization of viral sequences, coreceptor usage, and glycosylation. Proc. Natl Acad. Sci. USA 100, 12972-12977 (2003). 